\title{
Accelerated Publications
}

\section{Folding Intermediates of a $\beta$-Barrel Membrane Protein. Kinetic Evidence for a Multi-Step Membrane Insertion Mechanism ${ }^{\dagger, \dagger}$}

\author{
Jörg H. Kleinschmidt and Lukas K. Tamm* \\ Department of Molecular Physiology and Biological Physics, University of Virginia Health Sciences Center, P.O. Box 10011, \\ Charlottesville, Virginia 22906-0011
}

\begin{abstract}
The mechanism of folding and membrane insertion of integral membrane proteins, including helix bundle and $\beta$-barrel proteins is not well understood. A key question is whether folding and insertion are coupled or separable processes. We have used the $\beta$-barrel outer membrane protein A (OmpA) of Escherichia coli as a model to study the kinetics of folding and insertion into dioleoylphosphatidylcholine (DOPC) bilayers as a function of temperature by gel electrophoresis, protease digestion, and fluorescence spectroscopy. OmpA was unfolded in $8 \mathrm{M}$ urea solution (without detergent), and refolding and membrane insertion was initiated by rapid dilution of the urea concentration in the presence of phospholipid vesicles. In addition to the kinetically unresolved hydrophobic collapse in water, the time course of refolding of OmpA into DOPC bilayers exhibited three kinetic phases over a large temperature range. The first step was fast $\left(k_{1}=0.16 \mathrm{~min}^{-1}\right)$ and not very dependent on temperature. The second step was up to two orders of magnitude slower at low temperatures $\left(2{ }^{\circ} \mathrm{C}\right)$, but approached the rate of the first step at higher temperatures $\left(40^{\circ} \mathrm{C}\right)$. The activation energy for this process was $46 \pm 4 \mathrm{~kJ} / \mathrm{mol}$. A third slow process $\left(k_{3}=0.9 \times 10^{-2} \mathrm{~min}^{-1}\right.$ at $\left.40{ }^{\circ} \mathrm{C}\right)$ was observed at the higher temperatures. These results suggest that at least two membrane-bound intermediates exist when OmpA folds and inserts into lipid bilayers. We also show that both membrane-bound intermediates can be stabilized in fluid lipid bilayers at low temperatures. These intermediates share many properties with the adsorbed/partially inserted form of OmpA that was previously characterized in gel phase lipid bilayers [Rodionova et al. (1995) Biochemistry 34, 19211929]. Temperature jump experiments demonstrate, that the low-temperature intermediates can be rapidly converted to fully inserted native OmpA. On the basis of these and previous results, we present a simple folding model for $\beta$-barrel membrane proteins, in which folding and membrane insertion are coupled processes which involve at least four kinetically distinguishable steps.
\end{abstract}

In contrast to soluble proteins, the folding of membrane proteins has been studied only in rare cases. For example, bacteriorhodopsin (BR) ${ }^{1}$ can be refolded from a completely denatured state in detergent to a folded state in detergent/ lipid micelles (Huang et al., 1981; London \& Khorana, 1982).

\footnotetext{
$\dagger$ Supported by NIH grant AI30557. J.H.K. acknowledges a research stipend from the Deutsche Forschungsgemeinschaft.

$\$$ This paper is dedicated to the memory of Dr. Fritz Jähnig.

* Author to whom correspondence should be addressed. Tel: (804) 982-3578. FAX: (804) 982-1616. E-mail: 1kt2e@virginia.edu.

${ }^{\otimes}$ Abstract published in Advance ACS Abstracts, October 1, 1996.
}

Refolding experiments in detergents were also successful with the plant light-harvesting complex (LHC; Paulsen et

\footnotetext{
${ }^{1}$ Abbreviations: BR, bacteriorhodopsin; DMPC, 1,2-dimyristoyl$s n$-glycero-3-phosphocholine; DOPC, 1,2-dioleoyl-sn-glycero-3-phosphocholine; EDTA, ethylenediaminetetraacetic acid; LHC, lightharvesting complex; LUV, large unilamellar vesicles; OmpA, outer membrane protein A; PAGE, polyacrylamide gel electrophoresis; SDS, sodium dodecyl sulfate; SUV, small unilamellar vesicles; TLCK, $N^{\alpha}$ p-tosyl-L-lysine chloromethyl ketone; TM, transmembrane; TPCK, L-1tosylamide-2-phenylethyl chloromethyl ketone; Tris, (Tris[hydroxymethyl])aminomethane; Trp, tryptophan.
} 
al., 1990) and two $\beta$-barrel proteins of bacterial outer membranes, i.e., OmpA (Dornmair et al., 1990) and porin (Eisele \& Rosenbusch, 1990). Folding in lipid bilayers is likely different from folding in detergent micelles because the two model membranes have completely different geometries. Globular $\alpha$-helical membrane proteins are prohibitively hydrophobic to conduct refolding experiments in the absence of detergents, but OmpA and porin can be refolded and inserted into lipid bilayers from an unfolded state in urea (Surrey \& Jähnig, 1992; Surrey et al., 1996). This property makes these proteins excellent models to study protein folding and insertion into lipid bilayers. Although the kinetics of refolding were studied recently for four integral membrane proteins, namely, BR and LHC in detergent/lipid micelles (Booth et al., 1995; Booth \& Paulsen, 1996) and OmpA and porin in lipid vesicles (Surrey \& Jähnig, 1995; Surrey et al., 1996), no detailed mechanism of membrane protein folding has yet emerged from these studies.

A two-stage model has been proposed for the folding of helical membrane proteins (Popot \& Engelman, 1990). In this model, the membrane-spanning helices are considered to be independently stable structures, formed in stage I by membrane insertion and interacting in stage II to form the native tertiary structure. Evidence for this model comes, for example, from experiments in which fragments of BR or helical segments of the shaker $\mathrm{K}^{+}$channel were co-assembled within lipid bilayers (Kahn \& Engelman, 1992; Peled et al., 1996). Jacobs and White (1989) proposed a refined model for stage I helix insertion: hydrophobic polypeptide chain segments are thought to first bind to the membrane-water interface, helix formation occurs, and finally the TM helices penetrate the lipid bilayer. This last step must involve a rotation of the helix orientation and may be guided by parts of the sequence ("stays") that have a particularly high affinity for the interface. Good candidates for "stays" are the bulky aromatic residues, which in all known membrane protein structures (helical and $\beta$-barrels) are most frequently found near the bilayer-water interface (Schiffer et al., 1992; Cowan \& Rosenbusch, 1994). Recent Monte Carlo calculations support this model (Milik \& Skolnick, 1993, 1995). Also in agreement with the Jacobs and White model, helical secondary structure is induced in mitochondrial presequences at the membrane surface (Tamm, 1991) before these helices are translocated across the lipid bilayer (Maduke \& Roise, 1993). For the case of $\beta$-barrel proteins, it is not yet clear to what extent folding and insertion are two separable processes and whether the entire process can be subdivided into distinct stages similar to the two-stage model developed for helical membrane proteins.

OmpA provides a good model for studying the folding and membrane insertion of a $\beta$-barrel protein (Surrey \& Jähnig, 1992). The protein consists of 325 amino acids (Chen et al., 1980) with an apparent molecular mass of 35 $\mathrm{kDa}$ in the fully denatured state. The first $\sim 170$ residues form a membrane-inserted domain which spans the membrane with eight antiparallel $\beta$-strands (Cole et al., 1983; Morona et al., 1984; Freudl at al., 1986, 1989; Klose et al., 1986, 1993; Ried et al., 1994) in the form of a $\beta$-barrel
(Vogel \& Jähnig, 1986; Koebenik \& Krämer, 1995). ${ }^{2}$ The functions normally attributed to OmpA are maintenance of cell shape, facilitation of conjugation, and serving as a receptor for various phages (Chai \& Foulds, 1974; van Alphen, 1977; Sonntag et al., 1978). Recent studies have shown that OmpA binds to the surface of lipid bilayers in the gel phase and penetrates lipid bilayers in the liquidcrystalline phase (Surrey \& Jähnig, 1992; Rodionova et al., 1995). OmpA in its surface-bound form can be completely degraded by trypsin, while a $24 \mathrm{kDa}$ fragment remains protected from proteolysis when the protein is fully integrated into fluid lipid bilayers. The protein adopts a large amount of $\beta$-structure in both forms, i.e., $35-45 \%$ in the adsorbed state and $45-55 \%$ in the inserted native state (Rodionova et al., 1995). The five intrinsic tryptophans are about equally protected from aqueous quenchers of Trp fluorescence in both forms. Membrane-bound quenchers also quench the Trp fluorescence of OmpA: probes deep in the membrane are more effective in the adsorbed form, whereas probes near the membrane-water interface most efficiently quench native OmpA. This result suggests that OmpA in the adsorbed form partially penetrates the membrane or perturbs the lipid bilayer structure (Rodionova et al., 1995).

In a recent kinetic study of OmpA folding in lipid bilayers, a (single) membrane-bound intermediate has been identified (Surrey \& Jähnig, 1995). However, it is not clear from that work whether this intermediate has any relationship to the previously described adsorbed/partially inserted form of OmpA. In order to find evidence for early folding intermediates that may be stabilized at lower temperatures, we investigated in the present work the kinetics of OmpA refolding as a function of temperature by SDS-PAGE, proteolysis, and tryptophan fluorescence. SUVs of DOPC were chosen as target membranes because their low gel-toliquid-crystalline phase transition temperature $\left(T_{\mathrm{c}}=-18^{\circ} \mathrm{C}\right)$ allowed us to study the refolding kinetics in the fluid phase over a large temperature range. We present evidence for a new membrane-bound folding intermediate of OmpA and show that these intermediates which resemble the adsorbed/ partially inserted form are stabilized in fluid lipid bilayers at low temperatures. By performing temperature jump experiments, we further demonstrate that the low-temperature forms are readily converted to the inserted native state, suggesting that the adsorbed and partially inserted forms are true intermediates of OmpA folding and membrane insertion.

\section{MATERIALS AND METHODS}

Materials. OmpA was isolated and purified from the outer membrane of $E$. coli as described (Surrey \& Jähnig, 1992). DMPC and DOPC (Avanti Polar Lipids, Alabaster, AL) were checked by thin-layer chromatography and used without further purification. For folding experiments, a $10 \mathrm{mg} / \mathrm{mL}$ stock solution of OmpA in $8 \mathrm{M}$ urea was rapidly diluted into a urea-free solution of preformed vesicles.

Preparation of Small Unilamellar Vesicles. A $3 \mathrm{mg}$ amount of phospholipid was dissolved in chloroform, dried on the bottom of a glass test tube under a stream of nitrogen,

\footnotetext{
${ }^{2}$ On the basis of sequence homologies with other $\beta$-barrel membrane proteins and different computer algorithms, an alternative 16 stranded $\beta$-barrel structural model has been proposed for OmpA (Stathopoulos, 1996). The conclusions of the present paper do not depend on the number of strands in the $\beta$-barrel.
} 
and desiccated under high vacuum for at least $3 \mathrm{~h}$ to remove residual solvent. The lipids were dispersed in $10 \mathrm{mM}$ glycine buffer, pH 8.3, containing $1 \mathrm{mM}$ EDTA and $150 \mathrm{mM} \mathrm{NaCl}$, to a lipid concentration of $3 \mathrm{mg} / \mathrm{mL}$. SUVs were prepared by sonication of the lipid dispersion for $50 \mathrm{~min}$ using the microtip of a Branson ultrasonifier at $50 \%$ pulse cycle in an ice/water bath. Titanium dust was removed by centrifugation. All SUV preparations were equilibrated overnight at $4{ }^{\circ} \mathrm{C}$ and used on the day after preparation. Refolding and membrane insertion of OmpA into SUVs is much more efficient than into LUVs presumably due to a larger number of defect structures in highly curved membranes (Surrey \& Jähnig, 1992).

Folding and Membrane Insertion Detected by SDSPAGE. $9 \mu \mathrm{M}$ protein was incubated with a 400-fold molar excess of DOPC in $11 \mu \mathrm{L}$ of glycine buffer. Incubation was stopped by addition of $11 \mu \mathrm{L}$ of $0.125 \mathrm{M}$ Tris buffer, $\mathrm{pH}$ 6.8, containing 4\% SDS, $20 \%$ glycerol, and 10\% 2-mercaptoethanol at room temperature. For trypsin digestion experiments, samples were treated with trypsin (Sigma; type III, TPCK-treated) at a molar enzyme/substrate ratio of 200. Digestion was stopped with TLCK at a molar ratio of trypsin/ inhibitor of $1 / 250$. These samples were then treated with $11 \mu \mathrm{L}$ of $0.125 \mathrm{M}$ Tris buffer, $\mathrm{pH}$ 6.8, containing 4\% SDS, $20 \%$ glycerol, and $10 \%$ 2-mercaptoethanol at room temperature. SDS-PAGE was performed as described (Laemmli, 1970; Weber \& Osborne, 1964), but samples were not boiled in SDS.

Folding and Membrane Insertion Detected by Fluorescence Spectroscopy. All fluorescence experiments were carried out at a molar lipid/protein ratio of 400, with OmpA at a concentration of $1.4 \mu \mathrm{M}$ in a total volume of $1 \mathrm{~mL}$ of glycine buffer. Fluorescence measurements were performed on a SPEX Fluoromax spectrofluorimeter as described (Rodionova et al., 1995), except that an excitation bandwidth of $3 \mathrm{~nm}$ was selected. No photobleaching was observed during the entire time course of experiments under these conditions. The sample compartment was temperaturecontrolled, and the actual temperature of the sample was measured using a thermocouple and a digital thermometer (Omega, type HH21). Background spectra of the SUVs alone were taken first for later subtraction. $5 \mu \mathrm{L}$ of OmpA was then rapidly mixed into the cuvette at time zero, and OmpA spectra were recorded immediately (about $30 \mathrm{~s}$ after mixing) and after specified time intervals. The sample was not illuminated in between subsequent spectral recordings.

Temperature Jump Experiments. Spectra were taken as a function of time at the lower temperature as described above. After about 60-80 min they were transferred into a water bath at this same temperature, while the temperature of the sample compartment of the spectrofluorimeter was raised to the higher temperature. After this temperature was stable, the sample was transferred back into the spectrofluorimeter and the experiment was continued as before.

\section{RESULTS}

A Slow Folding Step Detected by SDS-PAGE and Tryptophan Fluorescence. Previous studies have shown that OmpA binds to lipid bilayers in two different forms (Surrey \& Jähnig, 1992; Rodionova et al., 1995). These two forms are distinguished by their relative mobility on SDS gels, if the samples are not boiled prior to loading the gel. The adsorbed/partially inserted form runs at an apparent molecular
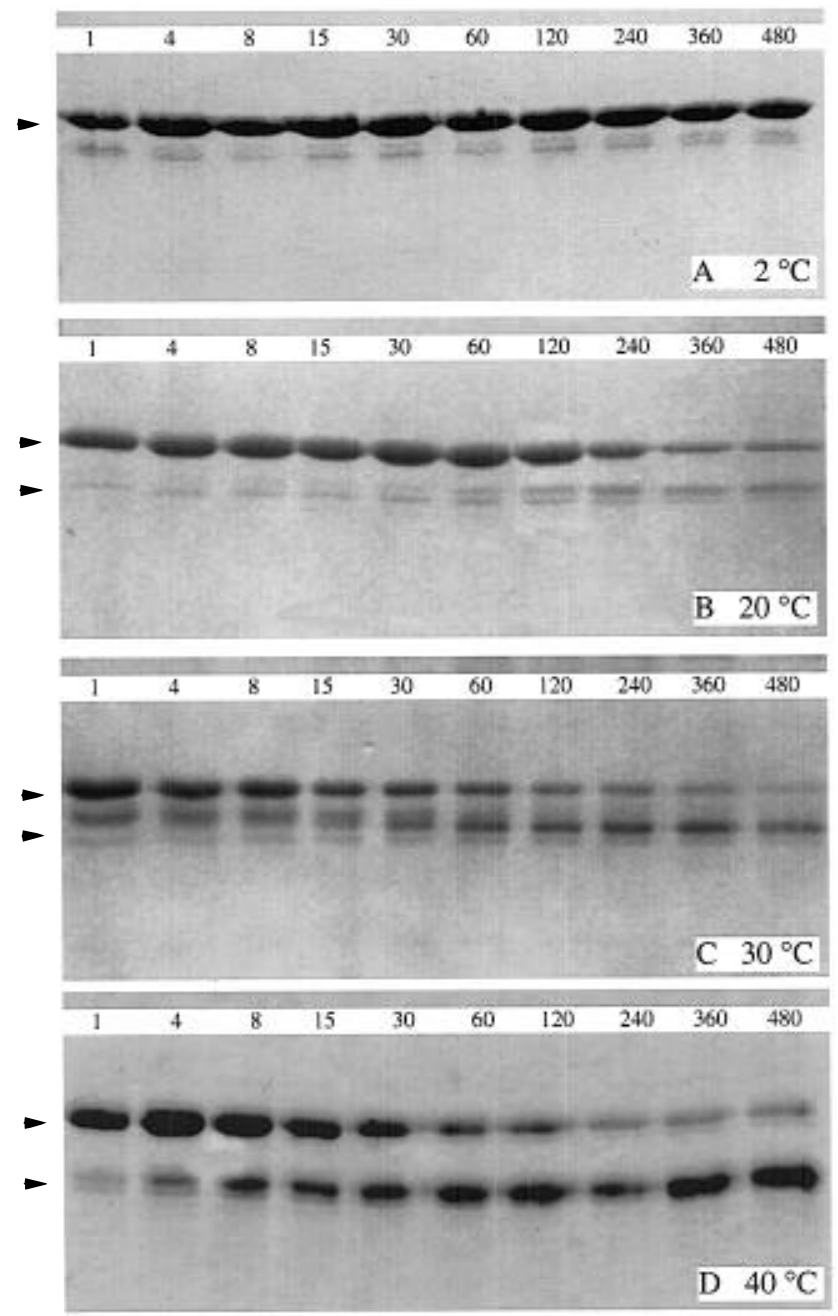

FIGURE 1: SDS-PAGE showing the time course of OmpA folding and insertion into SUV bilayers of DOPC at 2 (A), 20 (B), 30 (C), and $40{ }^{\circ} \mathrm{C}$ (D). $9 \mu \mathrm{M}$ OmpA was reacted with a 400 -fold molar excess of DOPC in glycine buffer $(10 \mathrm{mM}$, containing $1 \mathrm{mM}$ EDTA, $150 \mathrm{mM} \mathrm{NaCl}$ at $\mathrm{pH} \mathrm{8.3).} \mathrm{Incubations} \mathrm{were} \mathrm{stopped} \mathrm{by}$ addition of SDS. Samples were not boiled before loading onto the gels. The upper arrow in each gel indicates the membrane-adsorbed $35 \mathrm{kDa}$ protein, the lower arrow indicates the native $30 \mathrm{kDa}$ protein. Times are indicated in minutes.

mass of $35 \mathrm{kDa}$ and fully inserted native OmpA runs at 30 $\mathrm{kDa}$ under these conditions. The adsorbed form was previously only observed when OmpA was bound to DMPC or DPPC bilayers below their respective chain melting phase transitions. ${ }^{3}$ In order to study the kinetics of OmpA folding and insertion into fluid lipid bilayers over a large temperature range, we chose DOPC bilayers for these experiments. Figure 1 shows the time courses of OmpA folding in DOPC bilayers as detected by SDS-PAGE at various temperatures. At $2{ }^{\circ} \mathrm{C}$ OmpA remains in the $35 \mathrm{kDa}$ form for at least $8 \mathrm{~h}$. At $20{ }^{\circ} \mathrm{C} \mathrm{OmpA}$ folds and inserts into the membrane very slowly; $\sim 40 \%$ is converted to the $30 \mathrm{kDa}$ form after $8 \mathrm{~h}$. However, at higher temperatures, the $35 \mathrm{kDa}$ form gradually disappears and the $30 \mathrm{kDa}$ form appears, which becomes predominant after $60 \mathrm{~min}$ at $30{ }^{\circ} \mathrm{C}$ and $30 \mathrm{~min}$ at $40{ }^{\circ} \mathrm{C}$. The majority of the $35 \mathrm{kDa}$ form represents membrane-bound

\footnotetext{
${ }^{3}$ An indication that the adsorbed form may also exist in POPC bilayers at $4{ }^{\circ} \mathrm{C}$ was presented in Figure 1 of Rodionova et al. (1995), but this result was inconclusive since this temperature was close to the phase transition temperature of this lipid and the sample was incubated for only $2 \mathrm{~h}$.
} 


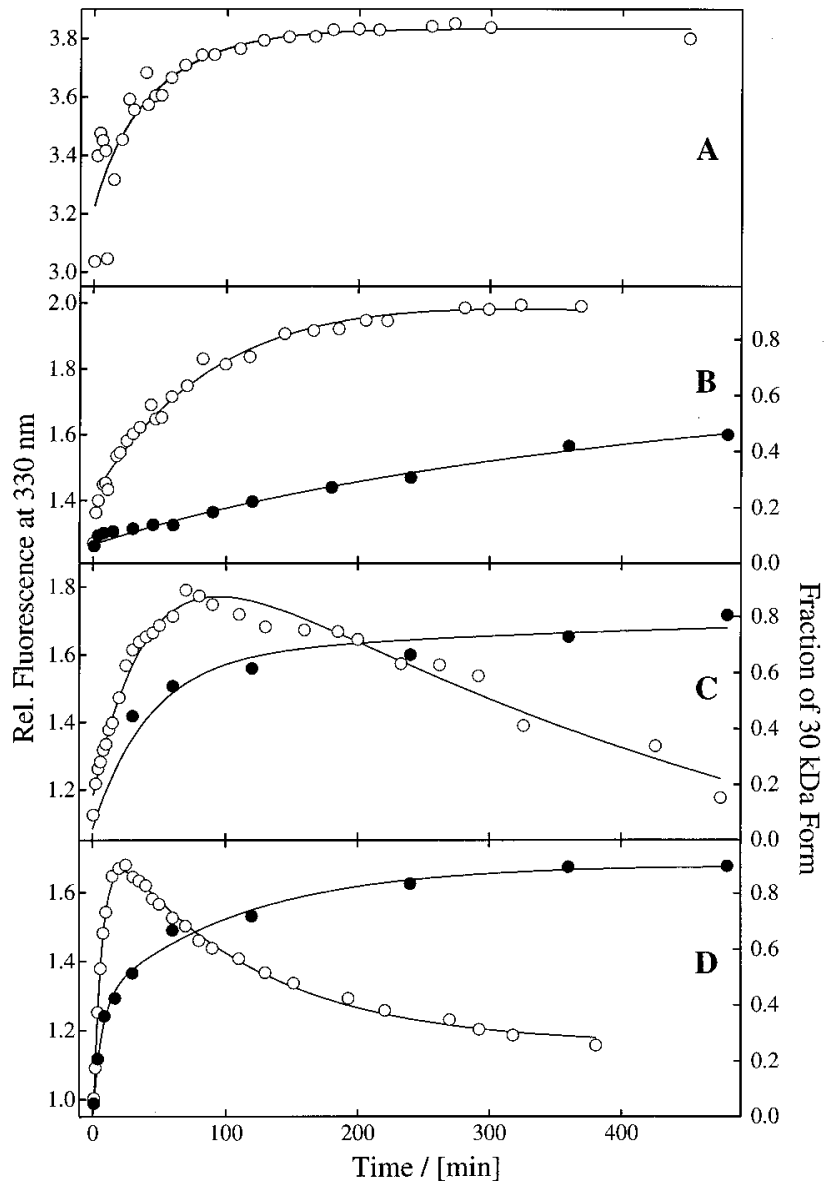

FIGURE 2: Kinetics of OmpA folding and membrane insertion as detected by the change of the fluorescence intensity at $330 \mathrm{~nm}(\mathrm{O})$ at 2 (A), 20 (B), $30(\mathrm{C})$, and $40{ }^{\circ} \mathrm{C}$ (D) and the formation of the native $30 \mathrm{kDa}$ form of OmpA (O). The fluorescence experiments were conducted at $1.4 \mu \mathrm{M}$ OmpA and a lipid/protein ratio of 400 . The relative fractions of $30 \mathrm{kDa} O \mathrm{OmpA}$ were quantified densitometrically from the gels of Figure 1.

and not completely unfolded or irreversibly aggregated protein because Trp fluorescence indicates membrane binding and because this form can be converted readily and quantitatively into the inserted native state after a temperature jump (see below). The $30 \mathrm{kDa}$ form is folded and fully membrane-inserted as can be demonstrated by the simultaneous appearance of the $24 \mathrm{kDa}$ membrane-protected fragment after trypsin digestion [data not shown, see also Surrey and Jähnig (1992) and Rodionova et al. (1995)]. (Note also that an as yet uncharacterized form of OmpA with an apparent molecular mass of $\sim 32 \mathrm{kDa}$ appears transiently on the $30{ }^{\circ} \mathrm{C}$ gel.)

OmpA contains five tryptophans which are all located in its membrane-inserted domain. We recorded Trp fluorescence spectra as a function of time and temperature using the same conditions as in the kinetic experiments of Figure 1. When OmpA interacts with lipid bilayers, the Trp fluorescence emission undergoes a blue shift and increases in intensity, i.e., a typical response to a more hydrophobic environment. The analysis of the time courses reveals that the blue shift and intensity change are not strictly coupled and follow different kinetics. Figure 2 shows a comparison of the time courses derived from densitometry of the gels of Figure 1 and the fluorescence intensity changes at $330 \mathrm{~nm}$. When not much native OmpA is formed at 2 and $20^{\circ} \mathrm{C}$, the intensity changes are well described with bi-exponential

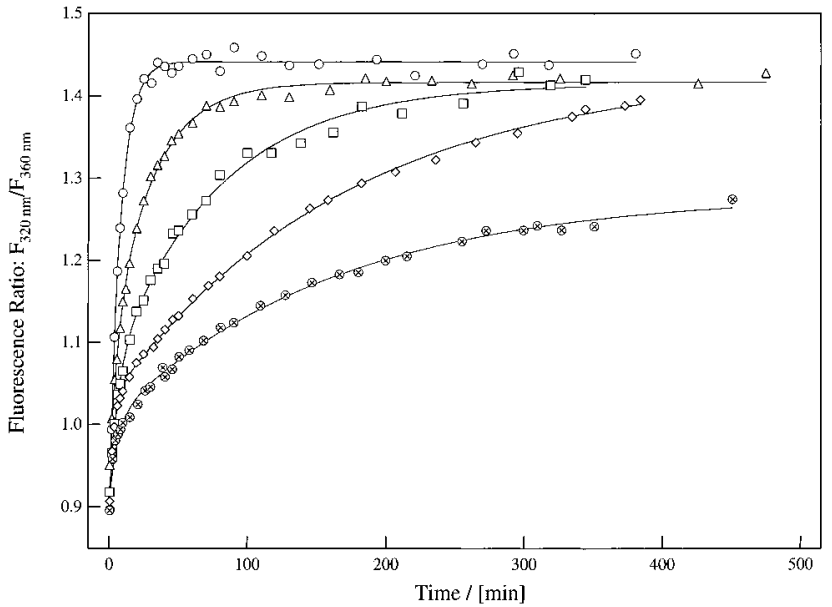

FIGURE 3: Fluorescence intensity ratios $F_{320 \mathrm{~nm}} / F_{360 \mathrm{~nm}}$ as a function of time showing the first two steps of OmpA folding and insertion into SUVs of DOPC. The temperatures were $2(\otimes), 10(\diamond), 20$ $(\square), 30(\triangle)$, and $40{ }^{\circ} \mathrm{C}(\bigcirc)$ and the conditions were the same as those of the fluorescence experiments of Figure 2. Each progress curve was fitted to a bi-exponential function in two separate rounds as described in the text. The best fit curves of the second round, in which the faster process was kept fixed at $k_{1}=0.16 \mathrm{~min}^{-1}$ are shown as solid lines. The rate constants of the slower process are those of Table 1, column 5 .

functions. However, at 30 and $40{ }^{\circ} \mathrm{C}$, three exponential processes, two with an increasing amplitude and one with a decreasing amplitude, are needed to fit the observed fluorescence intensity changes. The slowest process with a decreasing amplitude follows the same time course and yields essentially the same rate constant as the formation of the 30 $\mathrm{kDa}$ form on the SDS gels, i.e., $0.05 \times 10^{-2}$ and $0.9 \times 10^{-2}$ $\min ^{-1}$ at 30 and $40{ }^{\circ} \mathrm{C}$, respectively.

A Fast Adsorption and an Intermediate Step Detected by Tryptophan Fluorescence. For a detailed analysis of the kinetics of the interaction of OmpA with lipid bilayers, we plotted the ratio of the fluorescence intensities at $320 \mathrm{~nm}$ and $360 \mathrm{~nm}\left(F_{\mathrm{r}}=F_{320 \mathrm{~nm}} / F_{360 \mathrm{~nm}}\right)$ [for fluorescence spectra of OmpA under different conditions, see Figure 6 in Rodionova et al. (1995)]. We found that this parameter provides a sensitive and quantitative measure of the wavelength shifted species, independent of the absolute fluorescence intensity (see Discussion). Figure 3 shows the fluorescence ratio $F_{\mathrm{r}}$ as a function of time at various temperatures between 2 and $40{ }^{\circ} \mathrm{C}$. Analyzing $F_{\mathrm{r}}$ (or the blue shift) as a function of time allows us to separate the two faster processes from the slowest process that was described in the preceding section. The time courses of Figure 3 strongly depend on temperature, the slowest kinetics being observed at $2{ }^{\circ} \mathrm{C}$. At all temperatures the time courses of Figure 3 can be fit to bi-exponential functions. Since the two rate constants are nearly identical at $40{ }^{\circ} \mathrm{C}$, a monoexponential fit is sufficient in this case. Table 1 (columns 2 and 3) shows the rate constants derived from the biexponential fits of the data of Figure 3. The interaction of OmpA with DOPC is best described by a fast step occurring on a time scale of a few minutes and a second slower step with a time scale of about an hour. The fast step is relatively temperature-independent. Therefore, we used the average rate constant, $k_{1}=0.16 \mathrm{~min}^{-1}$, which was kept fixed in a second round of fitting to determine $k_{2}$. These rate constants are shown in columns 4 and 5 of Table 1, and the corresponding fits are presented as solid lines in Figures 3 
Table 1: Rate Constants Derived from the Kinetic Data of Figures 3 and $6^{a}$

\begin{tabular}{|c|c|c|c|c|}
\hline \multirow[b]{2}{*}{$\begin{array}{l}\text { temperature } \\
\left({ }^{\circ} \mathrm{C}\right)\end{array}$} & \multicolumn{2}{|c|}{$k_{1}$ and $k_{2}$ free } & \multicolumn{2}{|c|}{$k_{1}$ fixed, $k_{2}$ free } \\
\hline & $\begin{array}{c}\text { fast step } \\
k_{1} /\left(\min ^{-1}\right)\end{array}$ & $\begin{array}{c}\text { slow step } \\
k_{2} /\left(\mathrm{min}^{-1}\right)\end{array}$ & $\begin{array}{c}\text { fast step } \\
k_{1} /\left(\mathrm{min}^{-1}\right)\end{array}$ & $\begin{array}{c}\text { slow step } \\
k_{2} /\left(\min ^{-1}\right)\end{array}$ \\
\hline & & DOPC & \multirow{15}{*}{0.16} & \\
\hline 2 & 0.37 & $0.73 \times 10^{-2}$ & & $0.55 \times 10^{-2}$ \\
\hline 2 & 0.10 & $0.38 \times 10^{-2}$ & & $0.62 \times 10^{-2}$ \\
\hline 5 & 0.26 & $0.72 \times 10^{-2}$ & & $0.63 \times 10^{-2}$ \\
\hline 10 & 0.21 & $0.78 \times 10^{-2}$ & & $0.62 \times 10^{-2}$ \\
\hline 10 & 0.31 & $0.58 \times 10^{-2}$ & & $0.52 \times 10^{-2}$ \\
\hline 10 & 0.12 & $0.58 \times 10^{-2}$ & & $0.72 \times 10^{-2}$ \\
\hline 15 & 0.30 & $1.23 \times 10^{-2}$ & & $1.10 \times 10^{-2}$ \\
\hline 20 & 0.31 & $1.23 \times 10^{-2}$ & & $1.27 \times 10^{-2}$ \\
\hline 20 & 0.08 & $1.02 \times 10^{-2}$ & & $1.18 \times 10^{-2}$ \\
\hline 20 & 0.13 & $1.23 \times 10^{-2}$ & & $1.32 \times 10^{-2}$ \\
\hline 25 & 0.10 & $1.95 \times 10^{-2}$ & & $2.93 \times 10^{-2}$ \\
\hline 30 & 0.09 & $2.40 \times 10^{-2}$ & & $3.33 \times 10^{-2}$ \\
\hline 40 & 0.14 & $13.82 \times 10^{-2}$ & & $6.35 \times 10^{-2}$ \\
\hline 40 & 0.09 & $9.42 \times 10^{-2}$ & & $4.80 \times 10^{-2}$ \\
\hline & & DMPC & & \\
\hline 2 & 0.39 & $1.57 \times 10^{-2}$ & & \\
\hline 5 & 0.85 & $1.42 \times 10^{-2}$ & & \\
\hline 40 & 0.31 & $5.40 \times 10^{-2}$ & & \\
\hline
\end{tabular}

${ }^{a}$ Nonlinear least squares fits to a double exponential function were used to analyze the data. $k_{1}$ and $k_{2}$ were free fit parameters in a first round of fitting (columns 2 and 3 ). As the fast process was relatively temperature-independent, $k_{1}$ was kept fixed in a second round of fitting (columns 4 and 5).

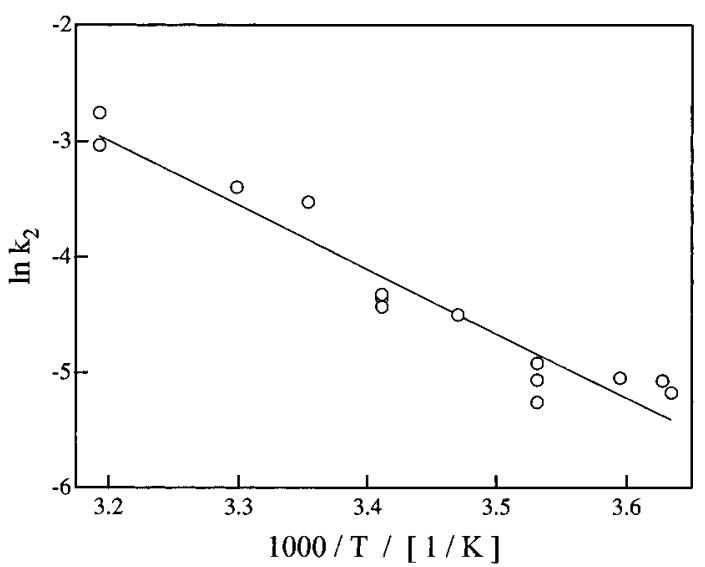

FIGURE 4: Arrhenius plot of the rate constants $k_{2}$ of OmpA refolding and membrane insertion. The activation energy of the process represented by $k_{2}$ was calculated to be $46.4 \pm 3.8 \mathrm{~kJ} / \mathrm{mol}$.

and 6. The agreement of this more restricted fit with the measured data is excellent. Moreover, these same rate constants were used as fixed parameters in the bi- and triexponential fits of the fluorescence data of Figure 2. Therefore, our kinetic analysis of all data of this work is completely self-consistent.

When $k_{2}$ is plotted as a function of temperature in an Arrhenius plot, a linear dependence is obtained (Figure 4). The activation energy of this process is $46.4 \pm 3.8 \mathrm{~kJ} / \mathrm{mol}$. A very similar activation energy $(54.7 \pm 6.2 \mathrm{~kJ} / \mathrm{mol})$ is obtained when the rate constants from the less restricted fits (column 3 of Table 1) are used, or when the blue shift is analyzed in a similar fashion $(43 \pm 4.7 \mathrm{~kJ} / \mathrm{mol})$. The similarity of the values obtained by the three methods of analysis confirm the general validity of our approach. We conclude from these data that binding, insertion, and folding of OmpA in bilayers of DOPC occurs in at least three kinetically resolvable steps. In addition, another (kinetically

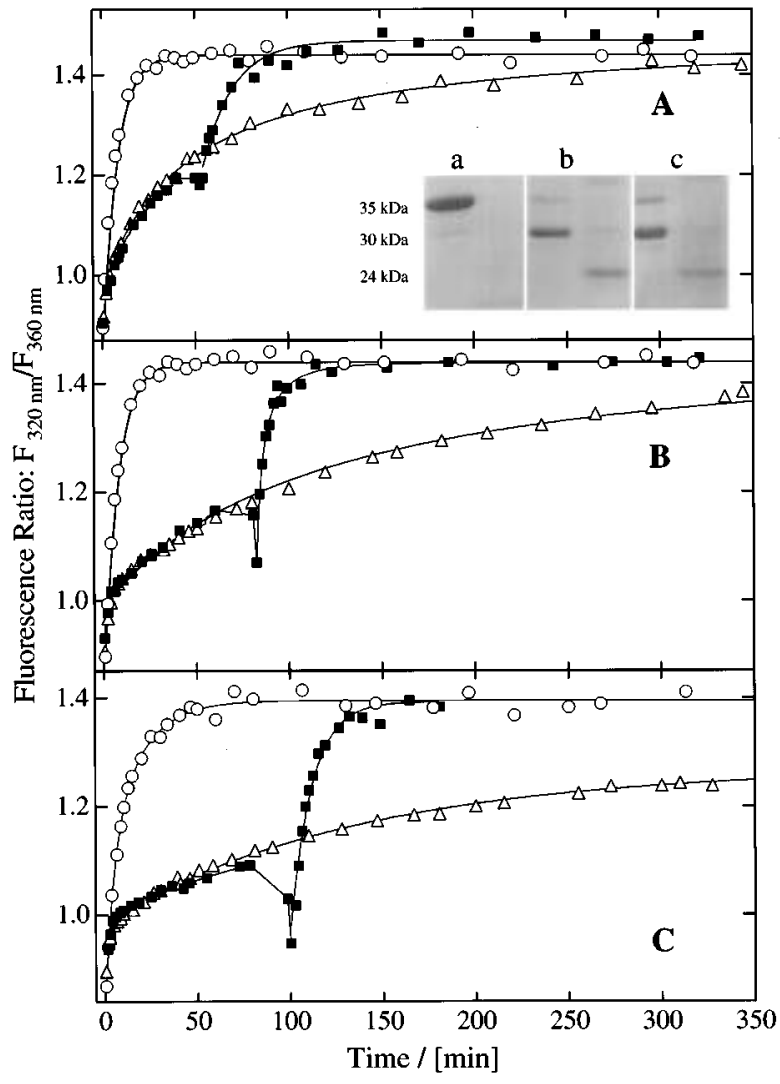

FIGURE 5: Response of the progress curves, $F_{\mathrm{r}}$ vs time, of the interaction of OmpA with SUVs of DOPC to a temperature jump. The samples were initially kept at lower temperatures [20 (A), 10 (B), and $\left.2{ }^{\circ} \mathrm{C}(\mathrm{C})\right]$ and then raised to $40{ }^{\circ} \mathrm{C}(\boldsymbol{\square})$. Isothermal experiments carried out at the corresponding individual temperatures are also shown for comparison $\left[\mathrm{O}, 40{ }^{\circ} \mathrm{C}\right.$ in all of the panels; $\triangle$, 20 (A), 10 (B), $\left.2^{\circ} \mathrm{C}(\mathrm{C})\right]$. The inset shows SDS-PAGE of refolding experiments at $4{ }^{\circ} \mathrm{C}(\mathrm{a}), 30^{\circ} \mathrm{C}(\mathrm{b})$, and after a temperature jump to $40{ }^{\circ} \mathrm{C}$, conducted $60 \mathrm{~min}$ after binding to SUVs at $4{ }^{\circ} \mathrm{C}$ (c). The samples in the right lanes of each panel were digested with trypsin.

unresolved) fast step occurs within the first $30 \mathrm{~s}$ of mixing of OmpA with DOPC vesicles, as the fluorescence ratio $F_{\mathrm{r}}$ increases rapidly from 0.44 to about 0.9 at all temperatures. This step is attributed to a fast hydrophobic collapse of OmpA without binding to the membrane (Surrey \& Jähnig, 1995).

Temperature Jump Experiments. To exclude that the intermediate forms of OmpA, which appear early after mixing with the lipid vesicles, are misfolded side products, we performed a series of temperature jump experiments. The reaction was started at a lower temperature, i.e., 2, 10, and $20{ }^{\circ} \mathrm{C}$, respectively, and then quickly raised to $40{ }^{\circ} \mathrm{C}$. The kinetic profiles of these experiments (Figure 5) show that the fluorescence ratios quickly adapt and precisely follow the kinetic profile at the higher temperature. As with the isothermal kinetic experiments, the temperature jump experiment was also reproduced when monitored by SDS-PAGE (Figure 5, inset), which proves that the slowest kinetic step is also completed after the temperature jump. These data strongly support the notion that the low-temperature forms are true intermediates in the folding process of OmpA.

Kinetics of Binding and Folding in DMPC Bilayers. For comparison, we also performed time-resolved folding experiments with SUVs of DMPC, a lipid with shorter, saturated acyl chains and a chain melting phase transition at $23{ }^{\circ} \mathrm{C}$. Figure 6 shows the data obtained at 5 and $40^{\circ} \mathrm{C}$, i.e., below 


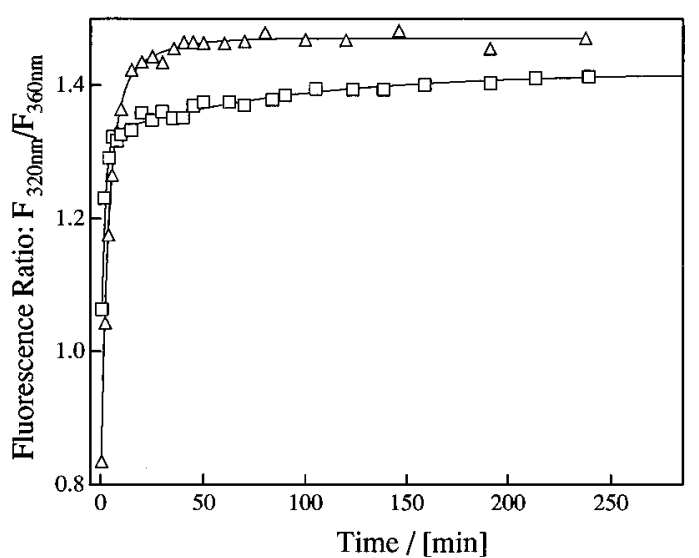

FIGURE 6: Fluorescence intensity ratios $F_{320 \mathrm{~nm}} / F_{360 \mathrm{~nm}}$ as a function of time after addition of $1.4 \mu \mathrm{M}$ OmpA to SUVs of DMPC at 5 $(\square)$ and $40{ }^{\circ} \mathrm{C}(\triangle)$. A lipid/protein ratio of 400 was used. The data were fitted to bi-exponential functions as described in the legend to Figure 3.

and above the phase transition temperature, respectively. Below the phase transition temperature, no native OmpA can be detected after several hours. Still, the kinetics are biphasic, suggesting that the two surface-bound (intermediate) forms exist even in gel phase lipid bilayers. Compared to DOPC, the bi-exponential fits yielded larger rate constants below and above $T_{\mathrm{c}}$, except for $k_{2}$ at $40{ }^{\circ} \mathrm{C}$ which was similar to $k_{2}$ observed with DOPC bilayers (Table 1).

\section{DISCUSSION}

A Stable Low-Temperature Intermediate of OmpA Folding in DOPC Bilayers. Our results show for the first time that an intermediate of OmpA folding can be stabilized in SUVs of DOPC at low temperatures. Below $\sim 10{ }^{\circ} \mathrm{C}$ the rate of protein insertion into these fluid bilayers in a completely folded form is so slow that it cannot be detected by SDSPAGE after 8 h. The increased and blue-shifted Trp fluorescence shows that the protein binds to lipid bilayers in about $6 \mathrm{~min}\left(k_{1}^{-1}\right)$ under these conditions. This form of OmpA can be completely digested by trypsin which indicates that it is not fully incorporated into the lipid bilayer. In this respect it resembles the form of OmpA that has been previously described in DMPC bilayers below the chain melting phase transition (Surrey \& Jähnig, 1992). This latter form of OmpA has been structurally characterized as membrane-adsorbed and partially penetrating by FTIR spectroscopy and fluorescence spectroscopy using an array of soluble and membrane-bound quenchers (Rodionova et al., 1995). The similar fluorescence properties, the similar mobilities on gels, and the similar rates of adsorption (Figure 6 and Table 1) strongly suggest that the dynamic structures of OmpA adsorbed to gel and fluid phase lipid bilayers at low temperatures are similar, but probably not identical as will be discussed further below. This form exhibits a significant amount of $\beta$-structure (Surrey \& Jähnig, 1992; Rodionova et al., 1995) which, however, is not yet organized into a $\beta$-barrel (Rodionova et al., 1995). The experiments of Figure 5 demonstrate that the low-temperature intermediate of OmpA is not an irreversible side product because it readily integrates into the membrane after a temperature jump.

Kinetics of OmpA Folding and Membrane Insertion. The second major new result of this work is the kinetic evidence for two membrane-bound folding intermediates of OmpA. Only one membrane-bound intermediate was detected in a previous kinetic analysis of OmpA insertion into DMPC/ DMPG bilayers at $30^{\circ} \mathrm{C}$ (Surrey \& Jähnig, 1995). At 40 ${ }^{\circ} \mathrm{C}$, we also find a degeneracy of the kinetic time course in DOPC bilayers where $k_{1}$ and $k_{2}$ become indistinguishable. At all other temperatures, three kinetic phases can clearly be distinguished (in addition to the kinetically unresolved hydrophobic collapse that occurs before membrane binding; Surrey \& Jähnig, 1995). We analyzed our data assuming first-order kinetics because protein insertion into membranes is better described by a partition than by a stoichiometric binding reaction when the lipids are in a large molar excess (Tamm, 1991) and because we found that self-association of OmpA is negligible during membrane binding and insertion (unpublished results). Analysis of the time courses detected by Trp fluorescence is greatly facilitated by separating the effect of lipid interactions on the wavelength shift or the ratio $F_{\mathrm{r}}=F_{320 \mathrm{~nm}} / F_{360 \mathrm{~nm}}$ and the fluorescence intensity $F_{330 \mathrm{~nm}}$. The first two parameters show the same biphasic kinetics (Figure 3), whereas $F_{330 \mathrm{~nm}}$ follows three exponential phases, the first two being identical to those also detected by the blue shift or $F_{\mathrm{r}}$. The third kinetic phase detected in the plots of $F_{330 \mathrm{~nm}}$ vs time is virtually identical to that detected by the shift of the relative mobility of the protein on the SDS gels (Figure 2) or the evolution of the membraneprotected $24 \mathrm{kDa}$ fragment after trypsin digestion (not shown). The fact that we observe the same rates by several different analytical procedures validates the employed methods of data analysis.

The first step $\left(k_{1}\right)$ was fast and essentially temperatureindependent. We attribute this step to the binding of OmpA to the bilayer surface [see above and Surrey and Jähnig (1995)]. The second step $\left(k_{2}\right)$ was strongly temperaturedependent (activation energy $46+4 \mathrm{~kJ} / \mathrm{mol}$ ) and ranged from minute $\left(\right.$ at $40{ }^{\circ} \mathrm{C}$ ) to hour (at $2-10{ }^{\circ} \mathrm{C}$ ) time scales. This strong temperature-dependence suggests that this process involves a major conformational change of the protein, perhaps accompanied by a partial insertion into the lipid bilayer, and/or a perturbation of the membrane lipids. The final step $\left(k_{3}\right)$ is very slow and also depends strongly on temperature. This step is not observed at temperatures below $10{ }^{\circ} \mathrm{C}$ (see above) and takes about $2 \mathrm{~h}$ at $40^{\circ} \mathrm{C}$. The protein becomes more compact during this process as is seen from its smaller apparent molecular mass on the SDS gels. Moreover, because it is transformed to a form in which its $\mathrm{N}$-terminal $24 \mathrm{kDa}$ domain is no longer digested by trypsin, OmpA is completely inserted into the membrane during this step in a form that is indistinguishable from its native state (Schweizer et al., 1978).

The major conclusions drawn from this kinetic analysis are summarized in Figure 7 in a tentative scheme for OmpA folding and membrane insertion which is an extension of the scheme presented by Surrey and Jähnig (1995). This simple sequential scheme is consistent with all available experimental data on OmpA folding and membrane insertion. However, the scheme is only tentative because more complex folding schemes cannot be excluded. According to this scheme, unfolded OmpA first collapses into a misfolded inside-out protein in aqueous solution $\left(\mathrm{I}_{\mathrm{W}}\right)$. $\mathrm{I}_{\mathrm{W}}$ then adsorbs to the membrane surface and forms the first membrane-bound intermediate $\left(\mathrm{I}_{\mathrm{M} 1}\right)$. The major difference between this and the earlier scheme of Surrey and Jähnig (1995) is that we 


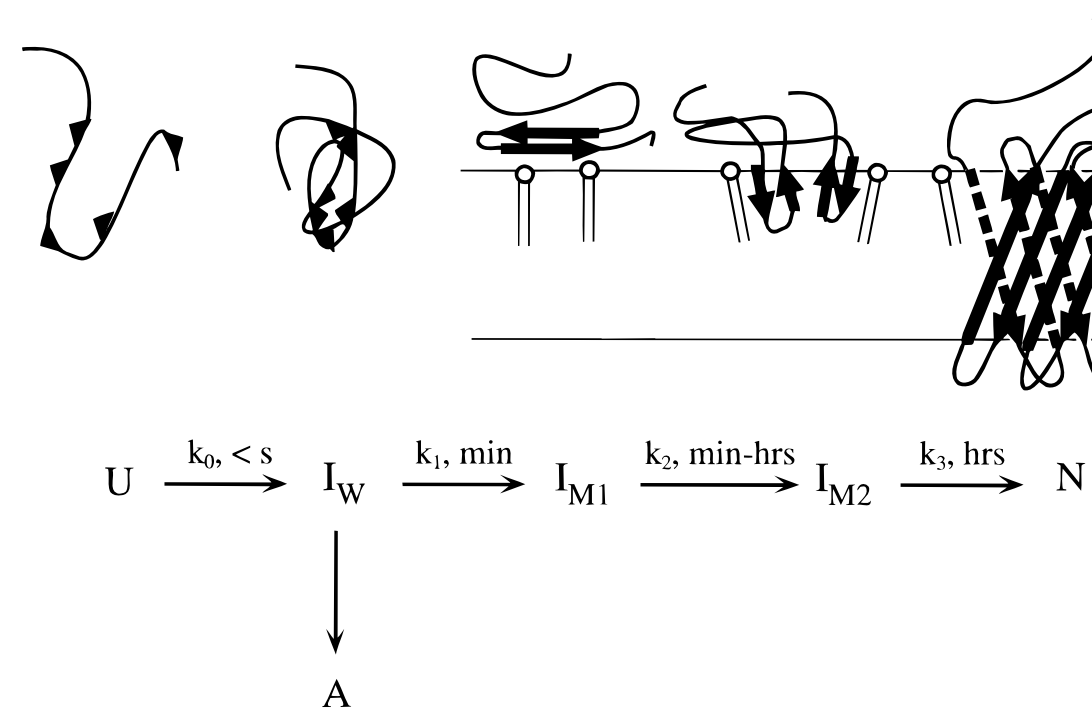

FIGURE 7: Tentative scheme for OmpA folding and membrane insertion. Unfolded OmpA (U) first hydrophobically collapses in a kinetically unresolved step to a water-soluble intermediate $\left(\mathrm{I}_{\mathrm{W}}\right)$ which then adsorbs to the lipid bilayer surface (intermediate $\left.\mathrm{I}_{\mathrm{M} 1}\right)$ while a minor fraction of the protein could possibly aggregate (A). The membrane-adsorbed intermediate progresses to a second membrane-bound intermediate $\left(\mathrm{I}_{\mathrm{M} 2}\right)$ before it is converted to the inserted, native state $(\mathrm{N})$.

have found evidence for a second membrane-bound, perhaps partially inserted folding intermediate of OmpA $\left(\mathrm{I}_{\mathrm{M} 2}\right)$. Given the similar adsorption kinetics, this form is likely similar to the one we have previously characterized in association with lipid bilayers in the gel phase (Rodionova et al., 1995). Both $\mathrm{I}_{\mathrm{M} 1}$ and $\mathrm{I}_{\mathrm{M} 2}$ are stabilized at low temperatures in DOPC, but because $k_{3}$ is the rate-limiting step in our folding scheme, $\mathrm{I}_{\mathrm{M} 2}$ becomes the predominant form after long incubation times. Since we have now found conditions to stabilize these intermediates and to vary their proportions as a function of the incubation time, it will be interesting to characterize their structures and biophysical properties in future experiments.

Fluorescence Intensity Ratios As a Measure of Kinetic Processes. We used the fluorescence intensity ratio $F_{\mathrm{r}}=$ $F_{320 \mathrm{~nm}} / F_{360 \mathrm{~nm}}$ as a function of time to separate the two faster processes from the slower third step, which affected only the absolute fluorescence intensity. A reaction composed of two consecutive irreversible steps is described by a biexponential kinetic progress curve. Wavelength shifts of peak fluorescence intensities may not be proportional to the concentrations of the species at either wavelength. However, the ratio of the fluorescence intensities at the peak wavelengths of the individual species is proportional to the ratio of the concentrations of the two species and is independent of changes of the absolute fluorescence intensities. When the solutions of the relevant rate equations of the scheme of Figure 7 are used to calculate $F_{\mathrm{r}}$, one finds an equation that depends on the ratio of the sums of two exponential functions in $k_{1}$ and $k_{2}$ and the differences of the fluorescence coefficients of the components at the two wavelengths. Inspection of fluorescence spectra taken under conditions where the individual forms predominate shows that the differences in the prefactors in the denominator are small compared to those in the numerator. In other words, the observed fluorescence intensity changes at $360 \mathrm{~nm}$ are small compared to those at $320 \mathrm{~nm}$ whenever the processes characterized by $k_{1}$ and $k_{2}$ dominate. This is the reason why we can quantitatively describe our experimental data of $F_{\mathrm{r}}$ vs time with two exponentials. A more detailed account of this analysis will be published elsewhere. An additional benefit of using fluorescence ratios is that individual kinetic experi- ments are normalized with respect to small differences in the actual protein concentration.

Unassisted Membrane Protein Folding Is Slow. The various steps of OmpA folding in membranes as observed in this work are slow compared to the elementary folding processes of soluble proteins (Kim \& Baldwin, 1990). This is not too surprising if one considers the high viscosity $(\sim 1$ poise) and the anisotropically ordered structure of the lipid bilayer. Lateral diffusion of the solvent molecules is two to three orders of magnitude slower in a membrane than in water and translocation of residues across the bilayer by simple diffusion may be even more restricted. Protein folding in bilayers as observed here in bilayers is also significantly slower than protein folding in detergent micelles (Booth et al., 1995; Booth \& Paulsen, 1996) where diffusional fluctuations may be intermediate between those of aqueous solutions and membranes. Binding and membrane insertion of membrane-interactive toxins is a process reminiscent of membrane protein folding because these proteins undergo large conformational rearrangements upon membrane insertion. Two characteristic times of the order of 10 s (adsorption) and a few min (hydrophobic insertion) were reported for the binding of the channel forming domain of colicin E1 to lipid bilayers (Shin et al., 1993). Again these processes are faster than refolding and membrane insertion of OmpA. In contrast to membrane protein folding studied here, toxins do not insert from a completely unfolded state; the major secondary structures are already formed in toxins and only a single helical hairpin is expected to insert into the bilayer which may explain the faster rates of insertion. In addition, the hairpin of toxins may not translocate completely across the membrane as suggested for colicin A (Lakey et al., 1993) which also binds to membranes in about $10 \mathrm{~s}$ at low pH (van der Goot et al., 1991).

Membrane insertion of outer membrane proteins must be much faster in vivo. Escherichia coli cells divide about once every $20 \mathrm{~min}$ at $37{ }^{\circ} \mathrm{C}$. Since OmpA is a major structural protein of the outer membrane, it must insert and fold at least as fast. Chaperonins speed up rates by preventing and correcting misfolded structures in the cytoplasm. For example, proOmpA is shielded from aggregation in the 
cytoplasm by SecB protein (Lecker et al., 1990). A candidate periplasmatic space chaperonin ( $\mathrm{Kkp}$ ) that binds OmpA and other outer membrane proteins has been identified and purified recently (Chen \& Henning, 1996). In addition, integral membrane proteins and lipopolysaccharides are likely to assist membrane insertion even though candidates for this specific role have yet to be identified in the outer membrane. Despite these differences between membrane protein folding in vivo and in vitro, studying and understanding this process in a well defined system is important because thermodynamically and kinetically accessible folding intermediates which likely are also present in vivo are more easily identified and biophysically characterized in the simpler systems. Finally, establishing methods for studying membrane protein folding in vitro may also help to determine the roles and mechanisms of chaperonins and other components that assist membrane protein folding under chemically and physically defined conditions.

\section{ACKNOWLEDGMENT}

We thank U. Henning for the gift of E. coli strain P400.6, F. Jähnig and P. Nollert (all Max-Planck Institut für Biologie, Tübingen, Germany) for their help with the purification of OmpA, and N. Rodionova (Mount Sinai Hospital, New York) for numerous helpful discussions.

\section{REFERENCES}

Booth, P. J., \& Paulsen, H. (1996) Biochemistry 35, 5103-5108.

Booth, P. J., Flitsch, S. L., Stern, L. J., Greenhalgh, D. A., Kim, P. S., \& Khorana, G. H. (1995) Struct. Biol. 2, 139-143.

Chai, T., \& Foulds, J. (1974) J. Mol. Biol. 85, 465-474.

Chen, R., \& Henning, U. (1996) Mol. Microbiol. 19, 1287-1294.

Chen, R., Schmidmayr, W., Krämer, C., Chen-Schmeisser, U., \& Henning, U. (1980) Proc. Natl. Acad. Sci. U.S.A. 77, 45924596.

Cole, S. T., Chen-Schmeisser, U., Hindennach, I., \& Henning, U. (1983) J. Bacteriol. 153, 581-587.

Cowan, S. W., \& Rosenbusch, J. P. (1994) Science 264, 914-916.

Dornmair, K., Kiefer, H., \& Jähnig, F. (1990) J. Biol. Chem. 265, 18907-18911.

Eisele, J.-L., \& Rosenbusch, J. P. (1990) J. Biol. Chem. 265, 10217-10220.

Freudl, R. (1989) Gene 82, 229-236.

Freudl, R., MacIntyre, S., Degen, M., \& Henning, U. (1986) J. Mol. Biol. 188, 491-494.

Huang, K.-S., Bayley, H., Liao, M.-J., London, E., \& Khorana, H. G. (1981) J. Biol. Chem. 256, 3802-3809.

Jacobs, R. E., \& White, S. H. (1989) Biochemistry 28, 3421-3437. Kahn, T. W., \& Engelman, D. M. (1992) Biochemistry 31:61446151.
Kim, P. S., \& Baldwin, R. L. (1990) Annu. Rev. Biochem. 59, 631660.

Klose, M., Störiko, A., Steirhof, Y.-D., Hindennach, I., Mutschler, B., \& Henning, U. (1993) J. Biol. Chem. 268, 25664-25670.

Klose, M., MacIntyre, S., Schwarz, H., \& Henning, U. (1986) J. Biol. Chem. 263, 13297-13302.

Koebnik, R., \& Krämer, L. (1995) J. Mol. Biol. 250, 617-626.

Laemmli, U. K. (1970) Nature 227, 680-685.

Lakey, J. H., Duché, D., González-Mañas, J.-M., Baty, D., \& Pattus, F. (1993) J. Mol. Biol. 230, 1055-1067.

Lecker, S. H., Driessen, A. J. M., \& Wickner, W. (1990) EMBO J. 9, 2309-2314.

London, E., \& Khorana, H. G. (1982) J. Biol. Chem. 257, 70037011.

Maduke, M., \& Roise, D. (1993) Science 260, 364-367.

Milik, M., \& Skolnick, J. (1993) Proteins: Struct., Funct., Genet. $15,10-25$.

Milik, M., \& Skolnick, J. (1995) Biophys. J. 69, 1382-1386.

Morona, R., Klose, M., Henning, U. (1984) J. Bacteriol. 159, 570578.

Paulsen, H., Rümler, U., \& Rüdiger, W. (1990) Planta 181, 204211.

Peled, H., Arkin, I. T., Engelman, D. M., \& Shai, Y. (1996) Biochemistry 35, 6828-6838.

Popot, J.-L., \& Engelman, D. M., (1990) Biochemistry 29, 40314037.

Ried, G., Koebnik, R., Hindennach, I., Mutschler, B. and Henning, U. (1994) Mol. Gen. Genet. 243, 127-135.

Rodionova, N. A., Tatulian, S. A., Surrey, T., Jähnig, F., \& Tamm, L. K. (1995) Biochemistry 34, 1921-1929.

Schiffer, M., Chang, C.-H., \& Stevens, F. J. (1992) Prot. Engr. 5, 213-214.

Schweizer, M., Hindennach, M., Garten, W., \& Henning, U. (1978) Eur. J. Biochem. 82, 211-217.

Shin, Y.-K., Levinthal, C., Levinthal, F., \& Hubbell, W. L. (1993) Science 259, 960-963.

Sonntag, I., Scharc, H., Hirota, Y., \& Henning, U. (1978) J. Bacteriol. 136, 280-285.

Stathopoulos, C. (1996) Protein Sci. 5, 170-173.

Surrey, T., \& Jähnig, F. (1992) Proc. Natl. Acad. Sci. U.S.A. 89, 7457-7461.

Surrey, T., \& Jähnig, F. (1995) J. Biol. Chem. 270, 28199-28203.

Surrey, T., Schmid, A., \& Jähnig, F. (1996) Biochemistry 35, $2283-$ 2288.

Tamm, L. K. (1991) Biochim. Biophys. Acta 1071, 123-148.

Van Alphen, L., Havekes, L., \& Lutgenberg, B. (1977) FEBS Lett. 75, 285-290.

Van der Goot, F. G., González-Mañas, J. M., Lakey, J. H., \& Pattus, F. (1991) Nature 354, 408-410.

Vogel, H., \& Jähnig, F. (1986) J. Mol. Biol. 190, 191-199.

Weber, K., \& Osborne, M. (1964) J. Biol. Chem. 244, 4406-4412. BI961478B 